\title{
Correlation and Path Coefficient Analysis for Grain Yield and Yield Components in Chickpea (Cicer arietinum L.) under Normal and Late Sown Conditions of Bihar, India
}

\author{
Tejashwini Agrawal ${ }^{1}$, Anand Kumar ${ }^{\text {* }}$, Sanjay Kumar ${ }^{1}$, Anil Kumar, \\ Ravi Ranjan Kumar ${ }^{2}$, Sanjeev Kumar ${ }^{3}$ and P.K. Singh ${ }^{1}$ \\ ${ }^{1}$ Department of Plant Breeding and Genetics, ${ }^{2}$ Department of Molecular Biology and Genetic \\ Engineering, ${ }^{3}$ Department of Plant Pathology, Bihar Agricultural University, \\ Sabour (Bhagalpur)-813210, India \\ *Corresponding author
}

\section{Keywords \\ Correlation, Path analysis, Grain yield, Chickpea (Cicer arietinum $\mathrm{L}$. )}

Article Info

Accepted:

15 January 2018 Available Online:

10 February 2018

\section{A B S T R A C T}

An experiment was conducted during Rabi, 2016-17 at Pulses Research farm, Bihar Agricultural University, Sabour (Bhagalpur), Bihar to study the correlation and path analysis for grain yield and its component characters in thirty six desi chickpea genotypes. The correlation study revealed that grain yield per plant was found significantly and positively correlated with number of primary branches, number of secondary branches, biological yield, harvest index, 100 seed weight and days to maturity, under normal sown condition. Under late sown condition grain yield per plant showed positive and significant association with chlorophyll index, number of primary branches, total number of pods per plant, effective pods per plant, biological yield, harvest index and 100 seed weight. Path analysis revealed that revealed that biological yield, effective pods per plant, harvest index, primary branches per plant and secondary branches per plant had high positive direct effect on grain yield per plant under normal condition while, chlorophyll index, effective pods per plant and 100 -seed weight would be quite effective in improving chickpea under late condition. On the basis of mean performance the genotypes viz. Phule G-13110, HI2-55, GNG 2215, GNG-469, GN 2213, DCP92-3 and BRC-1 were identified as the superior genotypes for yield and yield attributing traits under normal condition while, the genotypes viz. PhuleG-13110, HI2-55, BG 3043, NBeG 507 and ICCV 15112 were identified as superior genotypes under late sown condition. The present study has clearly indicated the need for giving due weight age to high chlorophyll index, number of primary branches, total number of pods per plant, effective pods per plant, high 100- seed and biological yield, harvest index and considered size of the seed and using these associated characters may be useful to the breeder to formulate appropriate breeding plans for selection of the genotype which tolerate high temperature condition while, under normal condition emphasis should be given on selection of characters viz. number of primary branches, number of secondary branches, biological yield and harvest index for improvement of grain yield. 


\section{Introduction}

Chickpea (Cicer arietinum L.), a member of Fabaceae family, is one of the most important rabi pulse crop in India contributing $20 \%$ to the pulse production in the world. Chickpea (Cicer arietinum L.) with a genome size of $732 \mathrm{Mbp}$ is a self-pollinated, diploid $(2 \mathrm{n}=2 \mathrm{x}$ $=16$ ) cool season pulse crop grown in more than 44 countries representing all the continents under eight geographically diverse agro-climatic conditions. India is the largest chickpea producer accounting a share of about $67 \%$ in global chickpea production with about $8.25 \mathrm{~m}$ ha area, $7.33 \mathrm{~m}$ t production and 889 $\mathrm{kg} / \mathrm{ha}$ productivity. The area occupied under chickpea cultivation in Bihar is 60 thousand hectares with a productivity of $958 \mathrm{~kg} / \mathrm{ha}$ (Project Coordinator Report, AICRP on chickpea, 2015-16).

Grain yield is a complex trait and highly influenced by many genetic and environmental factors. So, direct selection for yield as such could be misleading. A successful selection depends upon the information on the association of morphoagronomic traits with seed yield. The interrelationship between important yield components is best estimated by correlation coupled with path coefficient analysis. These techniques used in the breeding programme to exploit the yield potential for enhancing the productivity of chickpea and to develop high yielding improved varieties. Correlation is the mutual relationship between the variables, it aids in determining the most effective procedures for selection of superior genotypes. When there is positive correlation between major yield components, breeding strategies would be very effective but on the reverse, selection becomes very difficult. The estimates of correlation coefficients alone may be often misleading due to mutual cancellation of component characters. So, study of correlation coupled with a path analysis is more effective tool in the study of yield contributing characters. Path coefficient analysis is an important technique for partitioning the correlation coefficient into direct and indirect effect of the causal components on the complex component. Hence the present investigation was made to study the association between the characters and the direct and indirect effects of yield components on seed yield in chickpea.

\section{Materials and Methods}

The experimental material consisted of thirty six chickpea genotypes were sown on $10^{\text {th }}$ November under normal sown condition while, on $15^{\text {th }}$ December 2016 under late sown condition at Pulses Research farm, Bhitti, Bihar Agricultural University, Sabour (Bhagalpur). Atmospheric temperature varied from $4.0^{\circ} \mathrm{C}$ minimum in January to $45.0^{\circ} \mathrm{C}$ maximum in May. The experiment was laid out in a randomized complete block design with three replications during Rabi 2016-17 under All India Coordinated Research Project on Chickpea. The plot size was $4.8 \mathrm{~m}^{2}$, with 1 row of $4 \mathrm{~m}$ length. Inter row spacing distance was kept $30 \mathrm{~cm}$ and plant to plant spacing was $30 \times 10 \mathrm{~cm}$. The recommended packages of practices were followed to raise a healthy crop. Data were recorded on fifteen quantitative traits viz. days to $50 \%$ flowering, days to maturity, chlorophyll index, canopy temperature at vegetative stage, canopy temperature at reproductive stage, primary branches per plant, secondary branches per plant, plant height $(\mathrm{cm})$, total number of pods per plant, effective pods per plant, 100 seed weight $(\mathrm{g})$, grain yield per plant $(\mathrm{g})$, biological yield per plant, harvest index and grain yield per plot $(\mathrm{g})$. The days to $50 \%$ flowering, days to maturity, and grain yield per plot were recorded on a plot basis and plant height and number of pods per plant and 100-seed weight were recorded from a random sample of five plants in each plot. The genotypic and 
phenotypic correlation coefficients were computed using genotypic and phenotypic variances and co-variances (Al. Jibouri et al., 1958). The path coefficient analysis was done according to the method by Dewey and $\mathrm{Lu}$ (1959).

\section{Results and Discussion}

The analysis of variance showed significant differences among the genotypes (Table-1) for all the characters studied which provides an opportunity for selecting suitable genotypes with better performance for the traits. The study of inter-relationship among various characters in the form of correlation is, in fact, one of very important aspects in selection programme for the breeder to make an effective selection based on the correlated and uncorrelated response. Knowledge of nature and magnitude of associations among different characters are important on three counts. Indirect selection is important when desirable characters have low heritability measure in one sex only.

The efficiency of indirect selection is measured as a correlated response (Falconer, 1960). Knowledge of correlation is required when selection is to be made on several characters at a time through some simultaneous selection model. Even if, the objective is to make selection on a single trait, the knowledge of correlation is essential to avoid the undesirable correlated changes in other characters. In general, magnitude of genotypic correlation was higher than their corresponding phenotypic correlation coefficients in most of the characters suggesting that a strong inherent association exists for the traits studied and phenotypic selection may be rewarding. Similar results were also reported by Meena et al., (2006) and Kumar et al., (2017). Higher magnitude of genotypic correlation helps in selection for genetically controlled characters and give a better response for seed yield improvement than that would be expected on the basis of phenotypic association alone (Robinson et al., 1951).

The correlation analysis (Table-2) revealed that grain yield per plant was found significantly and positively correlated with number of primary branches, number of secondary branches, biological yield, harvest index,100- seed weight and days to maturity, in normal sown condition. Under late sown condition grain yield per plant showed positive and significant association with chlorophyll index, number of primary branches, total number of pods per plant, effective pods per plant, biological yield, harvest index and 100-seed weight. Similar finding were reported by Yadav and Haquae (2001), Sial et al., (2003), Talebi et al., (2007), Bicer and Sarkar (2008), Hahid et al., (2010), Ali et al., (2011), Babbar et al., (2012) and Kumar et al., (2017). Biological yield showed positive significant association with 100 seed weight $\left(0.355^{* *}\right)$ in normal sown condition and with harvest index $\left(0.234^{*}\right)$ and 100 seed weight $\left(0.641^{* *}\right)$ in late sown condition. Harvest index showed positive and significant association with 100 seed weight $\left(0.311^{* *}\right)$ under late sown condition in addition to others as mentioned above. A positive correlation between desirable traits is favourable to the plant breeder because it helps to bring the simultaneous improvement in both the characters. Similar findings for various traits were also reported by Yucel and Anlarsal (2010).

Total number of pods per plant showed positive significant association with number of effective pods per plant $\left(0.994^{* *}\right)$ and biological yield $\left(0.246^{*}\right)$ in normal sown condition and with number of effective pods per plant $\left(0.986^{* *}\right)$, biological yield $\left(0.775^{* *}\right)$, harvest index $\left(0.406^{* *}\right)$ and 100 seed weight $\left(0.338^{* *}\right)$ under late sown 
condition. Number of effective pods per plant showed positive significant association with number of biological yield $\left(0.232^{*}\right)$ in normal sown condition and with biological yield $\left(0.775^{* *}\right)$, harvest index $(0.400 * *)$ and 100 seed weight $\left(0.351^{* *}\right)$ under late sown condition. Days to $50 \%$ flowering showed positive significant association with days to maturity $\left(0.25^{*}\right)$ in normal sown condition. Under late sown condition, it showed positive significant correlation with days to maturity $\left(0.28^{*}\right)$, canopy temperature at pod filling stage $\left(0.425^{* *}\right)$ and plant height $\left(0.278^{*}\right)$ while significantly negative correlation was observed for chlorophyll index $(-0.37 * *)$. Days to maturity showed positive significant association with canopy temperature at vegetative $(0.250 *)$ and pod filling $(0.350 * *)$ stages in late sown condition and negative significant with chlorophyll index under normal sown conditions.

Chlorophyll index showed positive significant association with plant height $\left(0.249^{*}\right)$, primary branches $(0.232 *)$ and 100 seed weight $\left(0.426^{* *}\right)$ in normal sown condition and with canopy temperature at pod filling stage $\left(0.278^{*}\right)$, plant height $\left(0.303^{* *}\right)$, biological yield $\left(0.319^{* *}\right)$ and 100 seed weight $\left(0.270^{*}\right)$ in late sown condition. Canopy temperature at vegetative stage showed positive significant association only with number of secondary branches $\left(0.315^{* *}\right)$ under normal condition while, canopy temperature at pod filling stage showed positive significant association only with plant height $\left(0.460^{* *}\right)$ under late sown condition. Plant height showed positive significant association with number of primary branches per plant $\left(0.303^{* *}\right)$, biological yield $\left(0.238^{*}\right)$, harvest index $\left(0.455^{* *}\right)$ and 100 seed weight $(0.331 * *)$ in normal sown condition. Under late sown condition, it showed positive significant association with 100 seed weight $(0.251 *)$ while significant negative correlation with effective pods per plant $\left(-0.249^{*}\right)$.
Number of primary branches per plant showed positive significant association with number of secondary branches $(0.282 *)$ and biological yield $\left(0.529^{* *}\right)$ under normal condition while with secondary branches $\left(0.616^{* *}\right)$, number of total pods per plant $\left(0.575^{* *}\right)$, number of effective pods per plant $(0.582 * *)$, biological yield $\left(0.696^{* *}\right)$, harvest index $\left(0.279^{*}\right)$ and 100 seed weight $\left(0.530^{* *}\right)$ under late sown condition.

None of the traits were observed to be negatively and significantly associated with plant height in either of the environments. Number of secondary branches per plant showed positive significant association with biological yield $\left(0.277^{*}\right)$ under normal condition while with number of total pods per plant $\left(0.541^{* *}\right)$, number of effective pods per plant $(0.524 * *)$, biological yield $(0.592 * *)$ and 100 seed weight $(0.340 * *)$ under late sown condition. These results are agreement with the earlier reports of Priti et al., (2003). It means a slight increase in any one of the above traits may directly contribute towards seed yield.

The direct and indirect effects of investigated characters on grain yield at phenotypic level are presented in Table- 3 and path coefficient analysis revealed that biological yield, effective pods per plant, harvest index, primary branches per plant and secondary branches per plant had high positive direct effect on grain yield per plant under normal condition while, chlorophyll index, effective pods per plant and 100 -seed weight in late sown condition.

The earlier studies for direct effect on grain yield for biological yield and harvest index were reported by Babbar and Patel 2005, Kuldeep et al., 2014, Naveed et al., 2012 and Tadesse et al., 2016 and Kumar et al., 2017 whereas, Talebi et al., (2007), found positive indirect effect on seed yield. 
Table.2 Correlation coefficient analysis between yield and its component characters in thirty six chickpea genotypes under normal and late sown condition

\begin{tabular}{|c|c|c|c|c|c|c|c|c|c|c|c|c|c|c|}
\hline Characters & & 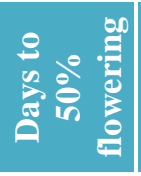 & 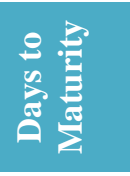 & $\begin{array}{l}\frac{\vec{z}}{0} \\
\overline{0} \\
\frac{0}{0} \\
\frac{0}{e} \\
\frac{0}{0}\end{array}$ & 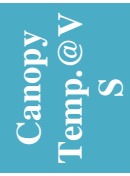 & 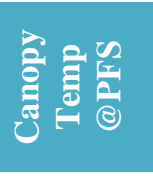 & 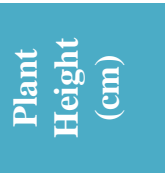 & 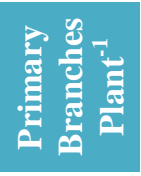 & 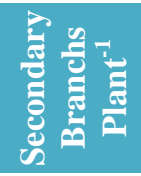 & 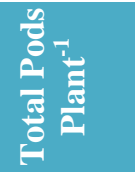 & 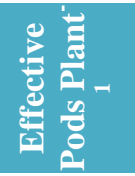 & 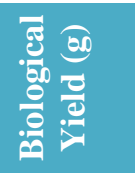 & 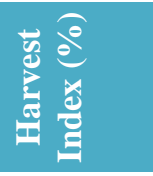 & 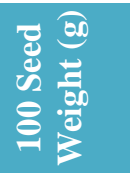 \\
\hline \multirow{2}{*}{$\begin{array}{l}\text { Days to } 50 \% \\
\text { Flowering }\end{array}$} & $\mathrm{N}$ & & $0.25^{*}$ & 0.00 & 0.014 & -0.039 & 0.168 & 0.074 & 0.153 & 0.193 & 0.193 & -0.016 & -0.002 & -0.164 \\
\hline & $\mathrm{L}$ & & $0.28 *$ & $-0.37 * *$ & 0.011 & $-0.425^{* *}$ & $0.278^{*}$ & 0.067 & -0.053 & -0.107 & -0.151 & -0.110 & 0.045 & 0.005 \\
\hline \multirow{2}{*}{$\begin{array}{l}\text { Days to } \\
\text { Maturity }\end{array}$} & $\mathrm{N}$ & & & $-0.25^{*}$ & 0.042 & 0.033 & -0.061 & -0.147 & 0.047 & 0.076 & 0.089 & 0.212 & 0.195 & 0.088 \\
\hline & $\mathrm{L}$ & & & 0.17 & $0.250 *$ & $-0.350 * *$ & 0.149 & 0.064 & -0.090 & -0.069 & -0.082 & 0.049 & -0.063 & 0.208 \\
\hline \multirow{2}{*}{$\begin{array}{l}\text { Chlorophyll } \\
\text { Index }\end{array}$} & $\mathrm{N}$ & & & & 0.004 & -0.071 & $0.249 *$ & $0.232 *$ & -0.080 & 0.090 & 0.098 & 0.136 & -0.153 & $0.426 * *$ \\
\hline & $\mathrm{L}$ & & & & 0.157 & $0.278^{*}$ & -0.197 & $0.303 * *$ & 0.019 & 0.085 & 0.134 & $0.319 *$ & -0.015 & $0.270^{*}$ \\
\hline \multirow{2}{*}{$\begin{array}{l}\text { Canopy } \\
\text { Temp.@vs } \\
\end{array}$} & $\mathrm{N}$ & & & & & -0.117 & -0.020 & -0.039 & $0.315 * *$ & 0.113 & 0.124 & -0.003 & -0.084 & -0.020 \\
\hline & $\mathrm{L}$ & & & & & -0.218 & 0.156 & 0.145 & 0.109 & -0.104 & -0.094 & 0.068 & -0.208 & 0.172 \\
\hline \multirow{2}{*}{$\begin{array}{l}\text { Canopy } \\
\text { Temp.@pfs } \\
\end{array}$} & $\mathrm{N}$ & & & & & & 0.052 & -0.105 & 0.015 & -0.067 & -0.074 & 0.050 & -0.102 & 0.004 \\
\hline & $\mathrm{L}$ & & & & & & $-0.460 * *$ & -0.080 & -0.073 & 0.143 & 0.196 & 0.059 & -0.059 & -0.192 \\
\hline \multirow[t]{2}{*}{ Plant Height cm } & $\mathrm{N}$ & & & & & & & $0.303 * *$ & 0.065 & 0.209 & 0.221 & $0.238 *$ & $-0.455^{* *}$ & $0.331 * *$ \\
\hline & $\mathrm{L}$ & & & & & & & 0.122 & 0.044 & -0.215 & $-0.249 *$ & -0.137 & 0.176 & $0.251 *$ \\
\hline \multirow{2}{*}{$\begin{array}{l}\text { Primary } \\
\text { Branches } \\
\text { Plant }^{-1}\end{array}$} & $\mathrm{~N}$ & & & & & & & & $0.282^{*}$ & 0.186 & 0.185 & $0.529 * *$ & -0.208 & 0.187 \\
\hline & $\mathrm{L}$ & & & & & & & & $0.616 * *$ & $0.575 * *$ & $0.582 * *$ & $0.696 * *$ & $0.279 *$ & $0.530 * *$ \\
\hline \multirow{2}{*}{$\begin{array}{l}\text { Secondary } \\
\text { Branches } \\
\text { Plant }^{-1} \\
\end{array}$} & $\mathrm{~N}$ & & & & & & & & & 0.031 & 0.032 & $0.277^{*}$ & -0.024 & 0.014 \\
\hline & $\mathrm{L}$ & & & & & & & & & $0.541 * *$ & $0.524 * *$ & $0.592 * *$ & 0.144 & $0.340 * *$ \\
\hline \multirow{2}{*}{$\begin{array}{l}\text { Total Pods } \\
\text { Plant }^{-1}\end{array}$} & $\mathrm{~N}$ & & & & & & & & & & $0.994 * *$ & $0.246^{*}$ & 0.119 & 0.125 \\
\hline & $\mathrm{L}$ & & & & & & & & & & $0.986 * *$ & $0.775^{* *}$ & $0.406 * *$ & $0.338 * *$ \\
\hline \multirow{2}{*}{$\begin{array}{l}\text { Effective Pods } \\
\text { Plant }^{-1}\end{array}$} & $\mathrm{~N}$ & & & & & & & & & & & $0.232 *$ & 0.104 & 0.131 \\
\hline & $\mathrm{L}$ & & & & & & & & & & & $0.775 * *$ & $0.400 * *$ & $0.351 * *$ \\
\hline \multirow{2}{*}{$\begin{array}{l}\text { Biological Yield } \\
(\mathrm{g})\end{array}$} & $\mathrm{N}$ & & & & & & & & & & & & 0.019 & $0.355^{* *} *$ \\
\hline & $\mathrm{L}$ & & & & & & & & & & & & $0.234^{*}$ & $0.641 * *$ \\
\hline \multirow{2}{*}{$\begin{array}{l}\text { Harvest Index } \\
(\%) \\
\end{array}$} & $\mathrm{N}$ & & & & & & & & & & & & & -0.136 \\
\hline & $\mathrm{L}$ & & & & & & & & & & & & & $0.311 * *$ \\
\hline \multirow[t]{2}{*}{ Seed index(g) } & $\mathrm{N}$ & & & & & & & & & & & & & \\
\hline & $\mathrm{L}$ & & & & & & & & & & & & & 1 \\
\hline \multirow{2}{*}{$\begin{array}{l}\text { Grain yield } \\
\text { plant }^{-1}\end{array}$} & $\mathrm{~N}$ & -0.059 & 0.228 & 0.099 & 0.024 & 0.018 & 0.091 & 0.461 & 0.289 & 0.186 & 0.180 & 0.939 & 0.250 & 0.286 \\
\hline & $\mathrm{L}$ & -0.033 & 0.080 & 0.370 & -0.016 & 0.028 & 0.040 & 0.403 & 0.222 & 0.395 & 0.407 & 0.423 & 0.264 & 0.399 \\
\hline
\end{tabular}


Table.3 Direct and indirect effect of different characters on grain yield in Chickpea under normal and late sown condition

\begin{tabular}{|c|c|c|c|c|c|c|c|c|c|c|c|c|c|c|}
\hline Characters & & 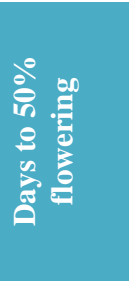 & 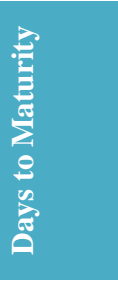 & 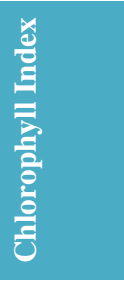 & 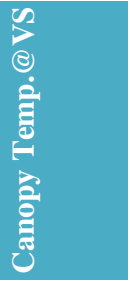 & 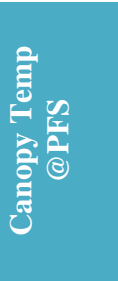 & 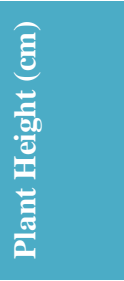 & 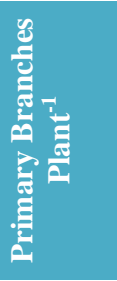 & 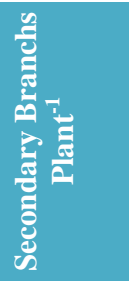 & 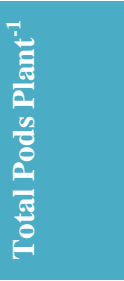 & 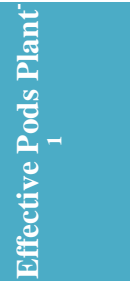 & 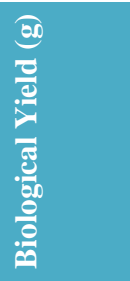 & 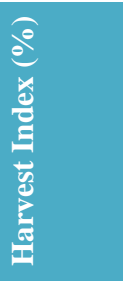 & 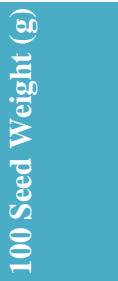 \\
\hline \multirow{2}{*}{$\begin{array}{l}\text { Days to } \mathbf{5 0 \%} \\
\text { Flowering }\end{array}$} & $\mathbf{N}$ & -0.032 & -0.008 & 0.000 & 0.000 & 0.001 & -0.005 & -0.002 & -0.005 & -0.006 & -0.006 & 0.001 & 0.000 & 0.005 \\
\hline & $\mathbf{L}$ & 0.120 & 0.034 & -0.045 & 0.001 & -0.051 & 0.033 & 0.008 & -0.006 & -0.013 & -0.018 & -0.013 & 0.005 & 0.001 \\
\hline \multirow[t]{2}{*}{ Days to Maturity } & $\mathbf{N}$ & -0.004 & -0.017 & 0.004 & -0.001 & -0.001 & 0.001 & 0.003 & -0.001 & -0.001 & -0.002 & -0.004 & -0.003 & -0.002 \\
\hline & $\mathbf{L}$ & -0.009 & -0.033 & -0.006 & -0.008 & 0.012 & -0.005 & -0.002 & 0.003 & 0.002 & 0.003 & -0.002 & 0.002 & -0.007 \\
\hline \multirow{2}{*}{ Chlorophyll Index } & $\mathbf{N}$ & 0.000 & -0.003 & 0.013 & 0.000 & -0.001 & 0.003 & 0.003 & -0.001 & 0.001 & 0.001 & 0.002 & -0.002 & 0.005 \\
\hline & $\mathbf{L}$ & -0.150 & 0.067 & 0.401 & 0.063 & 0.111 & -0.079 & 0.121 & 0.007 & 0.034 & 0.054 & 0.128 & -0.006 & 0.108 \\
\hline \multirow{2}{*}{$\begin{array}{l}\text { Canopy } \\
\text { Temp.@vs }\end{array}$} & $\mathbf{N}$ & 0.001 & 0.002 & 0.000 & 0.044 & -0.005 & -0.001 & -0.002 & 0.014 & 0.005 & 0.005 & 0.000 & -0.004 & -0.001 \\
\hline & $\mathbf{L}$ & -0.001 & -0.020 & -0.012 & -0.078 & 0.017 & -0.012 & -0.011 & -0.009 & 0.008 & 0.007 & -0.005 & 0.016 & -0.013 \\
\hline \multirow{2}{*}{$\begin{array}{l}\text { Canopy } \\
\text { Temp.@pfs }\end{array}$} & $\mathbf{N}$ & 0.000 & 0.000 & 0.000 & -0.001 & 0.006 & 0.000 & -0.001 & 0.000 & 0.000 & 0.000 & 0.000 & -0.001 & 0.000 \\
\hline & $\mathbf{L}$ & 0.013 & 0.011 & -0.009 & 0.007 & -0.032 & 0.015 & 0.003 & 0.002 & -0.005 & -0.006 & -0.002 & 0.002 & 0.006 \\
\hline \multirow[t]{2}{*}{ Plant Height (cm) } & $\mathbf{N}$ & -0.002 & 0.001 & -0.003 & 0.000 & -0.001 & -0.014 & -0.004 & -0.001 & -0.003 & -0.003 & -0.003 & 0.006 & -0.005 \\
\hline & $\mathbf{L}$ & 0.030 & 0.016 & -0.021 & 0.017 & -0.050 & 0.109 & 0.013 & 0.005 & -0.023 & -0.027 & -0.015 & 0.019 & 0.027 \\
\hline \multirow{2}{*}{$\begin{array}{l}\text { Primary } \\
\text { Branches/ Plant }\end{array}$} & $\mathbf{N}$ & 0.002 & -0.004 & 0.006 & -0.001 & -0.003 & 0.008 & 0.025 & 0.007 & 0.005 & 0.005 & 0.013 & -0.005 & 0.005 \\
\hline & $\mathbf{L}$ & 0.001 & 0.001 & 0.003 & 0.001 & -0.001 & 0.001 & 0.009 & 0.005 & 0.005 & 0.005 & 0.006 & 0.002 & 0.005 \\
\hline \multirow{2}{*}{$\begin{array}{l}\text { Secondary } \\
\text { Branches/ Plant }\end{array}$} & $\mathbf{N}$ & 0.003 & 0.001 & -0.001 & 0.005 & 0.000 & 0.001 & 0.005 & 0.017 & 0.001 & 0.001 & 0.005 & 0.000 & 0.000 \\
\hline & $\mathbf{L}$ & 0.000 & 0.000 & 0.000 & 0.000 & 0.000 & 0.000 & -0.001 & -0.002 & -0.001 & -0.001 & -0.001 & 0.000 & -0.001 \\
\hline \multirow{2}{*}{$\begin{array}{l}\text { Total number of } \\
\text { Pods/ Plant }\end{array}$} & $\mathbf{N}$ & -0.184 & -0.073 & -0.086 & -0.108 & 0.064 & -0.199 & -0.178 & -0.030 & -0.955 & -0.950 & -0.235 & -0.114 & -0.120 \\
\hline & $\mathbf{L}$ & -0.021 & -0.014 & 0.017 & -0.021 & 0.029 & -0.043 & 0.115 & 0.109 & 0.201 & 0.198 & 0.156 & 0.082 & 0.068 \\
\hline \multirow{2}{*}{$\begin{array}{l}\text { Effective Pods } \\
\text { /Plant }\end{array}$} & $\mathbf{N}$ & 0.170 & 0.078 & 0.086 & 0.109 & -0.065 & 0.195 & 0.164 & 0.029 & 0.877 & 0.882 & 0.205 & 0.091 & 0.115 \\
\hline & $\mathbf{L}$ & -0.032 & -0.017 & 0.028 & -0.020 & 0.041 & -0.053 & 0.123 & 0.111 & 0.208 & 0.211 & 0.164 & 0.085 & 0.074 \\
\hline \multirow{2}{*}{$\begin{array}{l}\text { Biological Yield } \\
\text { (g) }\end{array}$} & $\mathbf{N}$ & -0.015 & 0.203 & 0.131 & -0.003 & 0.048 & 0.228 & 0.507 & 0.266 & 0.236 & 0.223 & 0.960 & 0.019 & 0.341 \\
\hline & $\mathbf{L}$ & 0.014 & -0.006 & -0.041 & -0.009 & -0.008 & 0.018 & -0.090 & -0.077 & -0.101 & -0.101 & -0.130 & -0.030 & -0.083 \\
\hline \multirow[t]{2}{*}{ Harvest Index (\%) } & $\mathbf{N}$ & 0.000 & 0.050 & -0.040 & -0.022 & -0.026 & -0.118 & -0.054 & -0.006 & 0.031 & 0.027 & 0.005 & 0.259 & -0.035 \\
\hline & $\mathbf{L}$ & 0.001 & -0.001 & 0.000 & -0.005 & -0.001 & 0.004 & 0.006 & 0.003 & 0.009 & 0.009 & 0.005 & 0.023 & 0.007 \\
\hline \multirow[t]{2}{*}{ Seed index(g) } & $\mathbf{N}$ & 0.004 & -0.002 & -0.010 & 0.000 & 0.000 & -0.008 & -0.005 & 0.000 & -0.003 & -0.003 & -0.009 & 0.003 & -0.024 \\
\hline & $\mathbf{L}$ & 0.001 & 0.043 & 0.056 & 0.036 & -0.040 & 0.052 & 0.110 & 0.070 & 0.070 & 0.073 & 0.133 & 0.064 & 0.207 \\
\hline \multirow[t]{2}{*}{ Grain yield /plant } & $\mathbf{N}$ & -0.059 & 0.228 & 0.099 & 0.024 & 0.018 & 0.091 & 0.461 & 0.289 & 0.186 & 0.180 & 0.939 & 0.250 & 0.286 \\
\hline & $\mathbf{L}$ & -0.033 & 0.080 & 0.370 & -0.016 & 0.028 & 0.040 & 0.403 & 0.222 & 0.395 & 0.407 & 0.423 & 0.264 & 0.399 \\
\hline \multirow[t]{2}{*}{ Partial $\mathbf{R}^{2}$} & $\mathbf{N}$ & 0.002 & -0.004 & 0.001 & 0.001 & 0.000 & -0.001 & 0.011 & 0.005 & -0.178 & 0.158 & 0.901 & 0.065 & -0.007 \\
\hline & $\mathbf{L}$ & -0.004 & -0.003 & 0.148 & 0.001 & -0.001 & 0.004 & 0.004 & -0.001 & 0.079 & 0.086 & -0.055 & 0.006 & 0.083 \\
\hline
\end{tabular}


Table.1 Analysis of variance for fifteen quantitative characters of 36 chickpea genotypes under normal and late sown conditions

\begin{tabular}{|c|c|c|c|c|c|c|c|}
\hline \multirow[t]{3}{*}{ S. No. } & \multirow[t]{3}{*}{ CHARACTERS } & \multicolumn{6}{|c|}{ MEAN SUM OF SQUARES } \\
\hline & & \multicolumn{2}{|c|}{$\begin{array}{l}\text { REPLICATION } \\
\text { (d.f.=1) }\end{array}$} & \multicolumn{2}{|c|}{$\begin{array}{l}\text { TREATMENT } \\
(\text { d.f.=35) }\end{array}$} & \multicolumn{2}{|c|}{$\begin{array}{l}\text { ERROR } \\
(\text { d.f.=35) }\end{array}$} \\
\hline & & $\mathbf{N}$ & $\mathbf{L}$ & $\mathbf{N}$ & $\mathbf{L}$ & $\mathbf{N}$ & $\mathbf{L}$ \\
\hline 1 & Days to $50 \%$ flowering & 0.014 & 0.681 & $23.38^{* *}$ & $23.80^{* * *}$ & 9.56 & 6.45 \\
\hline 2 & Days to maturity & 9.389 & 6.125 & $19.34^{* *}$ & $4.48 * *$ & 4.65 & 1.56 \\
\hline 3 & Chlorophyll index & 9.329 & 0.025 & $19.34 *$ & $15.10 *$ & 9.14 & 8.04 \\
\hline 4 & Canopy temp.@VS & 2.029 & 0.016 & $3.22 *$ & 2.93* & 1.720 & 1.67 \\
\hline 5 & Canopy temp.@PFS & 4.093 & 2.286 & $6.82 *$ & $2.42 *$ & 3.48 & 1.16 \\
\hline 6 & Plant height $(\mathrm{cm})$ & 0.250 & 0.356 & $211.58 * *$ & $86.72^{* *}$ & 16.22 & 8.12 \\
\hline 7 & Primary branches & 0.142 & 0.002 & $0.27 * *$ & $0.33 * *$ & 0.034 & 0.03 \\
\hline 8 & Secondary branches & 0.020 & 0.405 & $1.56^{* *}$ & $1.47 * *$ & 0.34 & 0.35 \\
\hline 9 & Total pods per plant & 4.799 & 3.911 & $2011.73 * *$ & $403.35 * *$ & 101.17 & 25.5 \\
\hline 10 & Effective pods per plant & 2.534 & 1.237 & $2015.82 * *$ & $340.52 * *$ & 79.48 & 13.83 \\
\hline 11 & Biological yield (g) & 0.086 & 11.211 & $5508.29 * *$ & $174.99 * *$ & 179.74 & 8.05 \\
\hline 12 & Harvest index (\%) & 0.091 & 21.275 & $44.66^{* *}$ & $97.59 * *$ & 15.86 & 36.41 \\
\hline 13 & Seed index (g) & 2.170 & 0.586 & $110.37 * *$ & $86.46^{* *}$ & 1.21 & 0.74 \\
\hline 14 & Grain yield per plant (g) & 0.0361 & 3.849 & $342.21 * *$ & $42.60 * *$ & 8.31 & 1.08 \\
\hline 15 & Grain yield (kg/ha) & 86863.12 & 3096.47 & $410704.19^{* *}$ & $146866.58 * *$ & 54481.85 & 35022.62 \\
\hline
\end{tabular}

The result of association analysis indicated for improvement of chickpea grain yield per plant by selecting for number of primary branches, number of secondary branches, biological yield and harvest index in normal sown condition while, under late sown condition chlorophyll index, number of primary branches, total number of pods per plant, effective pods per plant, biological yield, harvest index and 100- seed weight. Days to $50 \%$ flowering had a negative direct effect on grain yield per plant, however an indirect positive effect was observed on grain yield per plant via canopy temperature at pod filling stage, biological yield and 100-seed weight and indirect negative effect via days to maturity, plant height, number of primary branches, number of secondary branches, total pods per plant and effective pods per plant under normal sown condition while, in late sown condition days to $50 \%$ flowering had positive direct effect on grain yield per plant however an indirect positive effect via days to maturity, canopy temperature at vegetative stage, plant height, number of primary branches harvest index and 100-seed weight and an indirect negative effect via chlorophyll index, canopy temperature at pod filling stage, number of secondary branches, total pods per plant, effective pods per plant and biological yield.

Days to maturity had a negative direct effect on grain yield per plant under both, normal and late sown conditions, however an indirect positive effect was observed on grain yield per plant via chlorophyll index, plant height and number of primary branches in normal sown condition whereas in late sown condition it was via canopy temperature at pod filling stage, number of secondary branches, total pods per plant, effective pods per plant and harvest index. Chlorophyll index had a positive direct effect on grain yield per plant under both, normal and late sown conditions however an indirect positive effect was observed on grain yield per plant via plant height, number of primary branches, total pods per plant, effective pods per plant, biological yield and 100-seed weight under 
normal condition and via days to maturity, canopy temperature at vegetative stage, canopy temperature at pod filling stage, number of primary branches, number of secondary branches, total pods per plant, effective pods per plant biological yield and 100-seed weight.

Canopy temperature at vegetative stage had a positive direct effect on grain yield per plant under normal sown condition. However an indirect positive effect was observed on grain yield per plant via days to $50 \%$ flowering, days to maturity, number of secondary branches per plant, total pods per plant and effective pods per plant. Under late sown condition, canopy temperature at vegetative stage had a negative direct effect on grain yield per plant while canopy temperature at pod filling stage, total pods per plant, and effective pods per plant and harvest index had a positive direct effect on grain yield. However an indirect positive effect was observed on grain yield per plant via remaining characters. Canopy temperature at pod filling stage had a positive direct effect on grain yield per plant under normal sown condition. However, an indirect negative effect was observed on grain yield per plant via canopy temperature at vegetative stage, number of primary branches and harvest index. Under late sown condition canopy temperature at pod filling stage had a negative direct effect on grain yield. However an indirect positive effect was observed via days to $50 \%$ flowering, days to maturity, canopy temperature at vegetative stage, plant height, number of primary branches, number of secondary branches, harvest index and 100seed weight. Plant height had a negative direct effect on grain yield per plant under normal condition. However an indirect positive effect was observed on grain yield per plant via days to maturity and harvest index in normal sown condition. Under late sown condition plant height had a positive direct effect on grain yield per plant. However an indirect positive effect was observed on grain yield per plant via days to $50 \%$ flowering, days to maturity, canopy temperature at vegetative stage, number of primary branches, number of secondary branches, harvest index and 100-seed weight.

Number of primary branches per plant had a positive direct effect on grain yield per plant under both conditions. However an indirect positive effect was observed on grain yield per plant via days to $50 \%$ flowering, chlorophyll index, plant height, number of secondary branches, total pods per plant, effective pods per plant, biological yield and 100 -seed weight in normal sown condition whereas, in late sown condition, an indirect positive effect was observed on grain yield per plant via all the characters except canopy temperature at pod filling stage. Number of secondary branches per plant had a positive direct effect on grain yield per plant under normal condition. However an indirect positive effect was observed on grain yield per plant via days to $50 \%$ flowering, days to maturity, canopy temperature at vegetative stage, plant height, number of primary branches, total pods per plant, effective pods per plant and biological yield whereas in late sown condition, a direct negative effect was observed accompanied with an indirect negative effect via number of primary branches, total pods per plant, effective pods per plant, biological yield and 100-seed weight.

Total pods per plant had a negative direct effect on grain yield per plant under normal condition. However an indirect positive effect was observed on grain yield per plant via canopy temperature at pod filling whereas in late sown condition total pods per plant had a positive direct effect on grain yield per plant. However an indirect positive effect was observed on grain yield per plant via 
chlorophyll index, canopy temperature at pod filling stage, number of primary branches per plant, number of secondary branches per plant, effective pods per plant, biological yield, harvest index and 100-seed weight. Effective pods per plant had a positive direct effect on grain yield per plant under both conditions. However an indirect positive effect was observed on grain yield per plant via all the characters except canopy temperature at pod filling whereas in late sown condition, an indirect positive effect was observed on grain yield per plant via chlorophyll index, canopy temperature at pod filling stage, number of primary branches per plant, number of secondary branches per plant, total pods per plant, biological yield, harvest index and 100-seed weight. Biological yield had a positive direct effect on grain yield per plant under normal sown condition. However an indirect positive effect was observed on grain yield per plant via all the characters except days to $50 \%$ flowering and canopy temperature at vegetative stage while in late sown condition, biological yield had a negative direct effect on grain yield per plant and indirect negative effect was through all the characters except days to $50 \%$ flowering and plant height. Harvest index had a positive direct effect on grain yield per plant under both conditions.

However an indirect positive effect was observed on grain yield per plant via days to maturity, total pods per plant, effective pods per plant, biological yield and 100-seed weight. In late sown condition however an indirect positive effect was observed on grain yield per plant via all the characters except days to maturity, canopy temperature at pod filling stage and plant height. 100 seed weight had a negative direct effect on grain yield per plant. However an indirect positive effect was observed on grain yield per plant via days to $50 \%$ flowering and harvest index while in late sown condition 100 seed weight had a negative direct effect on grain yield per plant. However an indirect positive effect was observed on grain yield per plant via all the characters except canopy temperature at pod filling stage.

On the basis of mean performance, the genotypes PhuleG-13110, HI2-55, GNG 2215, GNG-469, GN 2213, DCP92-3 and BRC-1were identified as the superior genotypes for yield and yield attributing traits viz. grain yield per plant, total number of pods per plant, effective pods per plant, primary branches per plant and 100-seed weight under normal condition while, the genotypes PhuleG-13110, HI2-55, BG 3043, NBeG 507 and ICCV 15112 under late sown condition.

Breeding strategies for improvement of yield potential in chickpea genotypes under heat stress would aim on selection of plants having high chlorophyll index, number of primary branches, total number of pods per plant, effective pods per plant, high 100- seed and biological yield, harvest index and considered size of the seed and using these associated characters may be useful to the breeder to formulate appropriate breeding plans for selection of the genotype which tolerate high temperature condition while, under normal condition emphasis should be given on selection of characters viz. number of primary branches, number of secondary branches, biological yield and harvest index for improvement of grain yield.

\section{References}

Ali, Q., Ahsan, M., Khaliq, I., Elahi, M., Shahbaz, M., Ahmed, W. and Naees, M. 2011. Estimation of genetic association of yield and quality traits in chickpea (Cicer arietinum L.). International Research Journal of Plant Science 2(6):166-169.

Al-Jibouri, H.A., Miller, P.A. and Robinson, H. F. 1958. Genotype and environmental 
variances and co-variance in upland cotton cross of interspecific origin. Agronomy J. 50: 633-637.

Babbar, A. and Patel, S.K. 2005. Correlation and path analysis in desi chickpea under Kymore Plateau Zone of Madhya Pradesh J.N.K.V. Res. J. 39(1): 47-51.

Babbar, A., Prakash, V., Prakash, T. and Iquabal, M.A. 2012. Genetic variability of chickpea (Cicer arietinum L.) under late sown condition. Legume Research 35(1): 1-7.

Bicer, B.T. and Sakar, D. 2008. Heritability and path analysis of some economical characteristics in lentil. Journal of Central Europe Agriculture 9(1): 191-196. Dewey, J.R. and Lu, K.H. 1959. A correlation and path coefficient analysis components of crested wheat grass seed production. Agronomy J. 51: 515-518.

Falconer, D.S. 1960. Introduction to Quantitative Genetics. Oliver and Boyd, Edinburgh pp. 365.

Hahid, S., Malik, R., Bakhsh, A., Asif, M.A., Iqbal, U. and Iqbal, S.M. 2010. Assessment of genetic variability and interrelationship among some agronomic traits in chickpea. International Journal of Agricultural Biology 12(1): 81-85.

Kuldeep, R.K., Pandey, S., Babbar, A. and Mishra, D.K. 2014. Genetic variability, character association and path coefficient analysis in chickpea grown under heat stress condition. Electronic Journal of Plant Breeding 5(4):812-819.

Meena, H.S., Kumar, J. and Deshmukh, P.S. 2006.Genetic variability and correlation studies for traits related to drought tolerance in chickpea (Cicer arietinum L.). Indian J. Genet. Pl. Breed. 66(2):140.

Naveed, M.T., Ali, Qurban, Ahsan, M. and
Hussain, B. 2012. Correlation and path coefficient analysis for various quantitative traits in Chickpea (Cicer arietinum L.). Int $\mathbf{J}$ for Agro Vet \& Med Sci, 6(2): 97-106.

Priti, G., Semwal, B.D., Gupta, D. 2003. Correlation and path analysis in black gram (Vigna mungo L. Hepper). Progressive Agriculture 3(12): 63- 65.

Robinson, H.F., Comstock, R.E., Harvey, P.H. 1951. Genotypic and phenotypic correlations in corn and their implications in selection. Agronomy Journal 43: 262267.

Sial, P., Mishra, P.K., Pathak, R.K. and Sial, M. 2003. Character association in chickpea. Environ. and Ecol. 21(3):675-679.

Tadesse M, Fikre A, Eshete M, Girman N, Korbun L, Mohamed R, Bekele D, Funga A and Ojiewo CO 2016. Correlation and path coefficient analysis for various quantitative traits in desi chickpea genotypes under rainfed conditions in Ethiopia. J of Agri Sci, 8(12):112.

Talebi, R., Fayaz, F. and Jelodar, N.A.B. 2007. Correlation and path coefficient analysis of yield and yield components of chickpea (Cicer arietinum L.) under dry land condition in the west of Iran. Asian Journal of Plant Science 6(7):1151-1154.

Yadav, N.P. and Haquae, M.F. 2001. Correlation and regression study of yield and yield components in Chickpea. J. Res. Agril. Univ. 11(4): 211-215.

Yucel, D.O. and Anlarsal, A.E. 2010. Determination of selection criteria with path analysis in chickpea breeding. Bulgarian J. Agril. Sci. 16(1): 42-48.

\section{How to cite this article:}

Tejashwini Agrawal, Anand Kumar, Sanjay Kumar, Anil Kumar, Ravi Ranjan Kumar, Sanjeev Kumar and Singh, P.K. 2018. Correlation and Path Coefficient Analysis for Grain Yield and Yield Components in Chickpea (Cicer arietinum L.) under Normal and Late Sown Conditions of Bihar, India. Int.J.Curr.Microbiol.App.Sci. 7(02): 1633-1642.

doi: https://doi.org/10.20546/ijcmas.2018.702.197 